\title{
High aspect ratio $(L / D)$ riser VIV prediction using wake oscillator model
}

\author{
Wan-Hai Xu, Xiao-Hui Zeng *, Ying-Xiang Wu \\ Institute of Mechanics, Chinese Academy of Sciences, Beijing 100190, China
}

\section{A R T I C L E I N F O}

\section{Article history:}

Received 6 December 2007

Accepted 27 August 2008

Available online 24 September 2008

Keywords:

Vortex-induced vibration

Riser

Aspect ratio

van der Pol equation

Wake oscillator

\begin{abstract}
A B S T R A C T
A two-dimensional (2-D) vortex-induced vibration (VIV) prediction model for high aspect ratio $(L / D)$ riser subjected to uniform and sheared flow is studied in this paper. The nonlinear structure equations are considered. The near wake dynamics describing the fluctuating nature of vortex shedding is modeled using classical van der Pol equation. A new approach was applied to calibrate the empirical parameters in the wake oscillator model. Compared the predicted results with the experimental data and computational fluid dynamic (CFD) results. Good agreements are observed. It can be concluded that the present model can be used as simple computational tool in predicting some aspects of VIV of long flexible structures.
\end{abstract}

(c) 2008 Elsevier Ltd. All rights reserved.

\section{Introduction}

Slender cylindrical structures, such as TLP tether, marine cables, and flexible risers, are widely applied in the offshore engineering. The vortex-induced vibration (VIV) is one of the important reasons that lead to the structural failure. Recently, offshore oil platforms have been installed in water depths of over $2000 \mathrm{~m}$. In such conditions, a better comprehension of the vortex dynamics causing vibration and fatigue of risers is essential.

With risers presenting such high aspect ratios $(L / D \sim$ 1000-4000) and complex flow fields around them, a complete three-dimensional (3-D) numerical simulation under realistic conditions is difficult. One practical approach to riser VIV predictions has been proposed in which the fluid flow solution is obtained on a series of two-dimensional (2-D) planes along the riser axis. A simple structural model based on Euler-Bernoulli beam theory is developed in order to accomplish the task of integrating the hydrodynamic forces calculation and the dynamic response of the riser. This method is appealing because it reduces a large 3-D computational fluid dynamic (CFD) problem to a large number of smaller 2-D problems. Furthermore, the method has had some success in comparison with experiments such as that reported by Yamamoto et al. (2004), Shulz and Meling (2004, 2005), Willden and Graham (2005). Holmes et al. (2006) and Constantinides et al. (2007) used fully 3-D CFD simulations coupled with relatively simple structural models to predict high aspect ratio riser VIV, achieved reasonably well results, and

\footnotetext{
* Corresponding author. Tel./fax: +861082544180.

E-mail address: zxh@imech.ac.cn (X.-H. Zeng).
}

demonstrated the possibility of calculating riser VIV using full 3-D CFD approach.

Model testing could give valuable insight in the phenomenon of VIV. Large-scale model testing of a tensioned steel riser $(L / D=3000)$ in well-defined sheared current was performed at Hanøytangen outside Bergen, Norway in 1997: see Huse et al. (1998).

Recently, experiments on long riser $(L / D=1407)$ have been conducted at Marintek's Ocean Basin in Trondheim, the riser was tested without VIV suppression and with various strake arrangements, the detail of the experiments were given by Trim et al. (2005). Model testing is generally a favorable approach to provide design data and verifications. However, it does have its limitations too, such as facility availability and capacity limits, model scale limit, difficulty of current profile generation, cost concerns, etc.

Therefore, for practical reasons, one cannot rely on CFD or experimental techniques for the long flexible structures VIV prediction, rather one needs to rely on reduced-order models that take into consideration all of the physical aspects, are validated by a combination of numerical simulation and experiments, and are capable of reliably predicting and simulating VIV. A considerable number of analytical and numerical models have been proposed to evaluate the flow response to the structure vibrations, including wake oscillator models. Hartlen and Currie (1970) were the first to propose the wake oscillator model. Their proposal was motivated by the suggestion of Bishop and Hassan (1964). Recently, the van der Pol oscillator was revisited for VIV prediction: Facchinetti et al. (2004a) verified the effect of the cylinder movement on the lift fluctuation via different type of coupling (displacement, velocity, and acceleration). They found that the optimal coupling term involves the body acceleration, rather than the displacement or velocity. Facchinetti et al. (2004b) 
extended the model to predict VIV and VIW (vortex induced waves) for cables and successfully predicted the experimental response behavior of a towed cable. Mathelin and de Langre (2005) pushed further Facchinetti's work to predict VIV of cables subjected to sheared flows. Violette et al. (2007) predicted the VIV of long structures using a wake oscillator model which was developed by Facchinetti et al. (2004a), compared the predicted results with DNS and experimental results. However, the model presented by Facchinetti et al. (2004a) fails in describing quantitatively the cylinder oscillation amplitude, and no relations have been developed between the model parameters in the model, which vary considerably from experiment to experiment (Skop and Griffin, 1973).

The structural response of the riser usually includes nonlinear geometric effects such as large displacements and rotations, etc. Especially, the nonlinear effects become more significant with increasing riser aspect ratio $(L / D)$. The presence of these effects means that a nonlinear structural model must eventually be incorporated in the solution of riser problems. But prior studies of VIV employ simple linear structure models with few exceptions, e.g., Lu and Dalton (1996) and Kim and Perkins (2002). The aim of the present work is to analyze the long riser VIV in uniform and sheared flow, a phenomenological model of the near wake based on van der Pol wake oscillator is used, a two dof nonlinear structural model, which ignored the in-line deflection and considered the nonlinear coupling between axial and cross-flow (CF) direction, is employed, as developed by Kuchnicki and Benaroya (2002). The coupling term of structure and wake oscillator is acceleration (Facchinetti et al., 2004a). The new empirical parameters in the wake oscillator model were calibrated and used here.

This paper is divided in four sections. Brief descriptions of the structural and wake oscillator model are given in the next section. In Section 3, comparation of the present model results is made with the published experimental data and CFD results. Finally, in the last section, conclusions are drawn based on the results presented.

\section{Model description}

\subsection{Structure model}

The tensioned vertical straight riser model, such as plotted in Fig. 1, is limited to CF and axial vibrations here. It has been proved that the nonlinear coupling between the axial and the CF motion becomes more significant with high aspect ratio; using the equations provided by Kuchnicki and Benaroya (2002), for convenience the effects of torsion and rotation are neglected:

$\rho A_{\mathrm{r}} \ddot{U}-\left(E A_{\mathrm{r}}\left(U^{\prime}+\frac{1}{2} V^{\prime 2}\right)\right)^{\prime}=F_{Z}$

$\left(\rho A_{r}+C_{A} \rho_{f} A_{f}\right) \ddot{V}-\left(E A_{r}\left(U^{\prime}+\frac{1}{2} V^{\prime 2}\right) V^{\prime}\right)^{\prime}+\left(E I V^{\prime \prime}\right)^{\prime \prime}+\frac{1}{2} C_{D} \rho_{f} D U_{\text {flow }} \dot{V}=F_{Y}$

Note that prime notation is used for the derivative with respect to $Z$, and dot notation with respect to $T$, where $\rho$ is the density of the structure, $A_{\mathrm{r}}$ the cross-sectional area, $E$ the modulus of elasticity, $I$ the moment of inertia, $U$ the displacement of the structure in the $Z$ direction and $V$ in the $y$ direction, $F_{Z}$ the external forcing function in the $Z$ direction and $F_{Y}$ the forcing term due to the effects of vortices onto the structure in the $Y$ direction, $D$ the diameter of structure, $\rho_{\mathrm{f}}$ the density of the surrounding fluid, $A_{\mathrm{f}}$ the crosssection of the displaced volume, $C_{\mathrm{A}}$ the added mass coefficient, $C_{\mathrm{D}}$ the drag coefficient, $U_{\text {flow }}$ the flow velocity.

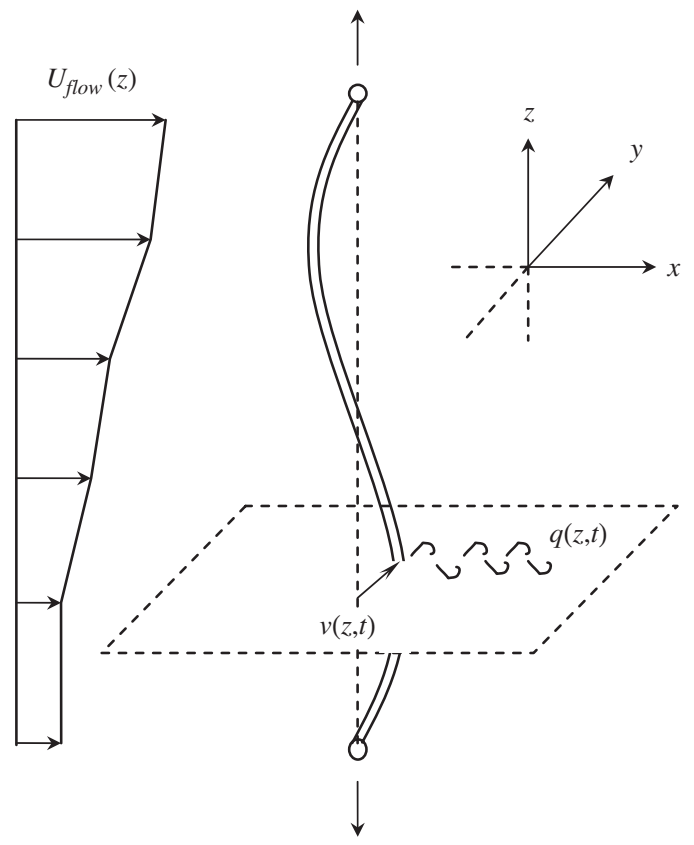

Fig. 1. High aspect ratio structure under a depth-varying flow.

\subsection{Wake model}

A forced van der Pol oscillator equation is used to describe the dynamics of the cylinder's wake (Nayfeh, 1993)

$\ddot{q}+\varepsilon \Omega_{\mathrm{f}}^{2}\left(q^{2}-1\right) \dot{q}+\Omega_{\mathrm{f}}^{2} q=F_{\mathrm{d}}$

The terms $\varepsilon$ is damping terms (Hartlen and Currie, 1970), it was taken as being related to the structural damping factor, the mass ratio (Skop and Griffin, 1973). The variable $q$ is defined as the local fluctuating lift coefficient, $q=2 C_{\mathrm{L}} / C_{\mathrm{LO}}, C_{\mathrm{LO}}$ is the reference lift coefficient of a fixed structure subjected to vortex shedding, $C_{\mathrm{L}}$ the lift coefficient. $F_{\mathrm{d}}$ the forcing term due to the action of the structure onto the fluid and $\Omega_{\mathrm{f}}$ the incident flow pulsation depending on the local fluid velocity through the Strouhal law

$\Omega_{\mathrm{f}}=2 \pi \mathrm{St} \frac{U_{\text {flow }}}{D}$

\subsection{Coupling of wake and structure}

As in the reference (Facchinetti et al., 2004a), the forcing term on the structure due to the wake dynamics in Eq. (2) is assumed to be

$F_{Y}=\frac{1}{2} \rho_{\mathrm{f}} U_{\text {flow }}^{2} D \frac{C_{\mathrm{L} 0}}{2} q$

The forcing on the wake due to the cylinder motion in Eq. (3) has been evaluated by Facchinetti et al. (2004a), using experimental data of VIV of elementary systems (one degree of freedom) in uniform flow. It could be found that the acceleration of the structure as the forcing term on fluid agreed good with some aspects of VIV observed experimentally for rigid cylinders elastically supported. Thus the coupling term in Eq. (3) is expressed as

$F_{\mathrm{d}}=\frac{A}{D} \ddot{V}$

where $A$ is the scaling parameter. 


\subsection{Calibration of the model parameters}

All parameters presented above are estimated in this section, assuming $\mathrm{St}=0.2, C_{\mathrm{L} 0}=0.3$ in the sub-critical range, 300 $<\operatorname{Re}<1.5 \times 10^{5}$ (Blevins, 1990). Drag coefficient $C_{\mathrm{D}}$ taken equal to 1.2 (Yamamoto et al., 2004; Violette et al., 2007), for a circular cylinder, added mass coefficient $C_{\mathrm{A}}=1.0$ (Blevins, 1990).

The value of $A$ as well as that of $\varepsilon$ was determined from the experimental results on forced and free VIV. Firstly introducing the dimensionless time $t=T \Omega_{\mathrm{f}}$ and space coordinate $y=Y / D$. The dimensionless displacement $v$ in the CF direction is $v=V / D$. Eq. (3) is now expressed in dimensionless form as

$\ddot{q}+\varepsilon\left(q^{2}-1\right) \dot{q}+q=A \ddot{v}$

Considering an harmonic motion of dimensionless amplitude $v_{0}$ and angular frequency $\omega$, namely $v=v_{0} \cos (\omega t)$. A reduced velocity was defined based on the forcing frequency as $U_{\mathrm{r}}=$ $1 /(\omega S t)$ (Facchinetti et al., 2004a), enforcing the hypothesis of harmonicity and frequency synchronization, the response is sought in the form $q=q_{0} \cos (\omega t-\psi)$, where $q_{0}$ and $\psi$ are timeindependent amplitude and phase, respectively. Substituting in the wake oscillator, Eq. (7), and considering only the main harmonic contribution of the nonlinearities, elementary algebra yields the amplitude of the transfer function of the wake oscillator, which has the same form as shown by Facchinetti et al. (2004a):

$q_{0}^{6}-8 q_{0}^{4}+16\left[1+\left(\frac{\omega^{2}-1}{\varepsilon \omega}\right)^{2}\right] q_{0}^{2}=16\left(\frac{A \omega^{2} v_{0}}{\varepsilon \omega}\right)^{2}$

The free wake oscillator response $q_{0}=2$ is supposed to prevail on the forced response, defining a lock-out state, looking for the boundary from polynomial $(8)$ in the $\left(U_{\mathrm{r}}, v_{0}\right)$ plane, the lock-in domain is shown in Fig. 2, the parameter $A$ may now be chosen by matching the model response (8) to experimental data on lock-in extension in the literature, it can be expressed as

$A=4$ for $0 \leqslant U_{\mathrm{r}} \leqslant 5 \quad A=10$ for $U_{\mathrm{r}}>5$

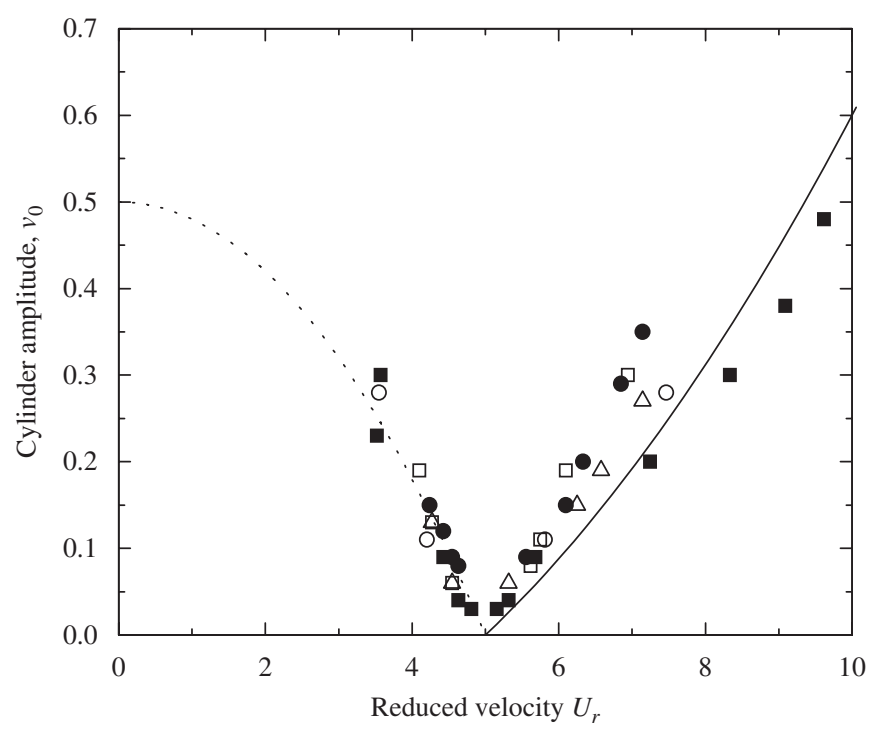

Fig. 2. Lock-in bands in the $\left(U_{\mathrm{r}}, v_{0}\right)$ plane for synchronization of vortex shedding with transverse cylinder vibration (Blevins, 1990). Experimental data: Koopman (1967), $\square$ for $\operatorname{Re}=100$, $\bullet$ for $\operatorname{Re}=200$, and $\triangle$ for $\operatorname{Re}=300$; Stansby (1976). for $\operatorname{Re}=3600$, $\circ$ for $\operatorname{Re}=9200$. Model parameters:,$- A=10 ; . ., A=4$.
For a given set of model parameters, the amplitude of cylinder vibration is a function of the structural damping factor, $\xi$, the mass ratio, $\mu$, and the ratio of the nominal vortex shedding frequency to the natural frequency of the cylinder structure. However, the maximum structure displacement amplitude at lock-in is typically expressed in the previous literature as a function of a single combined mass-damping parameter, namely the Skop-Griffin parameter $S_{\mathrm{G}}\left(=2 \pi^{3} \mathrm{St}^{2} \mu \xi\right)$. The maximum structure displacement amplitude can be written as (Facchinetti et al., 2004a)

$v_{0 \max }=\frac{C_{\mathrm{L} 0} / 2}{S_{\mathrm{G}}+\pi^{3} \mathrm{St}^{2} \gamma} \sqrt{1+\frac{A}{\varepsilon} \frac{C_{\mathrm{L} 0} / 4}{S_{\mathrm{G}}+\pi^{3} \mathrm{St}^{2} \gamma}}$

where $\gamma$ is a stall parameter (Balasubramanian and Skop, 1997; Skop and Luo, 2001), it can be expressed as $C_{\mathrm{D}} /\left(\pi^{2} \mathrm{St}\right)$. Assumed that the maximum structure displacement amplitude is the same as the experimental results presented by Iwan (1975) and Benassai and Campanile (2002)

$v_{0 \max }=\frac{1}{1+9.6\left(S_{\mathrm{G}} / 2 \pi \mathrm{St}^{2}\right)^{1.8}}$

The direct substitution of Eqs. (9) and (11) into Eq. (10) gives the specification of the empirical parameter $\varepsilon$. It can be seen that, $\varepsilon$ depends on the material properties of the structure, instead of being universal constant. The coupled Eqs. (1), (2) and (3) are solved numerically in space and time. The numerical method remains the same as used by Facchinetti et al. (2004b) where a centered finite differences scheme was applied both in time and space.

\section{Comparison with experimental data and CFD results}

In this section, a series of calculations were performed as direct simulation of the experiments in uniform flow (Trim et al., 2005) and sheared flow (Lie and Kaasen, 2006), respectively, these simulation could give a good picture of the successes or failures of the wake oscillator model described above. Comparisons of the calculations employing wake oscillator model with experiments (Trim et al., 2005; Lie and Kaasen, 2006) and CFD method (Holmes et al., 2006; Constantinides et al., 2007) are carried out. The focus is on evaluating the capability of the wake oscillator to reproduce some predictions from CFD models. A zero transverse displacement and bending moment conditions are imposed on the boundaries for the riser, and the initial axial displacement could be estimated using $u_{0}=N_{0} L / E A_{\mathrm{r}}$, where $N_{0}$ is the initial tension. A random noise of order $O\left(10^{-3}\right)$ amplitude is applied on the fluid variable $q$ as initial conditions. The first time derivative of the fluid variable is set to zero at $T=0$. Zero velocity are posed for the riser at $T=0$ (Violette et al., 2007).

Table 1

Physical property of risers in uniform flow Baarholm et al. (2005)

\begin{tabular}{ll}
\hline Total length & $38.0 \mathrm{~m}$ \\
Outer diameter & $0.027 \mathrm{~m}$ \\
Inner diameter & $0.024 \mathrm{~m}$ \\
Bending stiffness & $37.2 \mathrm{Nm}^{2}$ \\
Axial stiffness & $5.09 \times 10^{5} \mathrm{~N}$ \\
Mass (water filled) & $0.933 \mathrm{~kg} / \mathrm{m}$ \\
Mass (air filled) & $0.761 \mathrm{~kg} / \mathrm{m}$ \\
Mass ratio & 1.62 \\
Aspect ratio & 1407 \\
Tension at top & $4-6 \mathrm{kN}$
\end{tabular}




\subsection{Uniform flow}

The experiments described by Trim et al. (2005) were performed in a tow tank that provided well controlled flow conditions. The physical properties of the riser models are given in Table 1 (Baarholm et al., 2005). In our calculation the tension within the riser is set to $5 \mathrm{kN}$, while in the model testing it varies in a range from 4 to $6 \mathrm{kN}$, the aspect ratio $L / D$ is 1407 . In all the experiments, the riser is pinned at the ends and either towed by the two ends to simulate uniform current conditions as illustrated in Fig. 3. The experimental data consisted of several tow speeds ranging from 0.3 to $2.4 \mathrm{~m} / \mathrm{s}$. Here we only use the bare riser experimental data published by Trim et al. (2005) for comparisons.

Holmes et al. (2006) and Constantinides et al. (2007) attempted to use fully 3-D CFD simulations coupled with relatively simple structural models to predict riser VIV. This approach bypasses the main objections to the strip method but introduces new difficulties associated with the size of the numerical solution. To avoid excessive computational time and storage space, a number of compromises are made in order to keep the size of the problem tractable. In particular, the fluid mesh is made very coarse. The CFD results have been compared to the experimental data described by Trim et al. (2005), general agreements were observed.

The results of present simulations were compared with experimental data and CFD results by comparing dominant predicted and observed modes, dimensionless root mean square (RMS) amplitude of vibration along the length of the riser and mean maximum RMS amplitude from all points on the riser. Fig. 4 compares the predicted results of the riser CF motion RMS amplitude by wake oscillator model with experimental data (Trim et al., 2005) and CFD results (Holmes et al., 2006) at uniform flow speed of $0.4 \mathrm{~m} / \mathrm{s}$. It can be seen that wake oscillator model predicted the third mode is dominant. Also the model testing and CFD method (Holmes et al., 2006) show the dominant mode is third. The maximum amplitude by experiment is larger than CFD results and nearly the same as present predicted results for

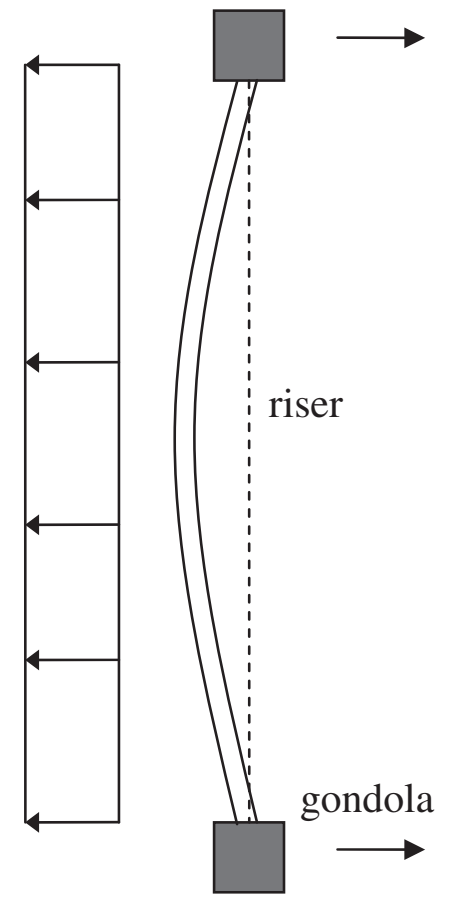

Fig. 3. Test schematics (plan view) in uniform flow.

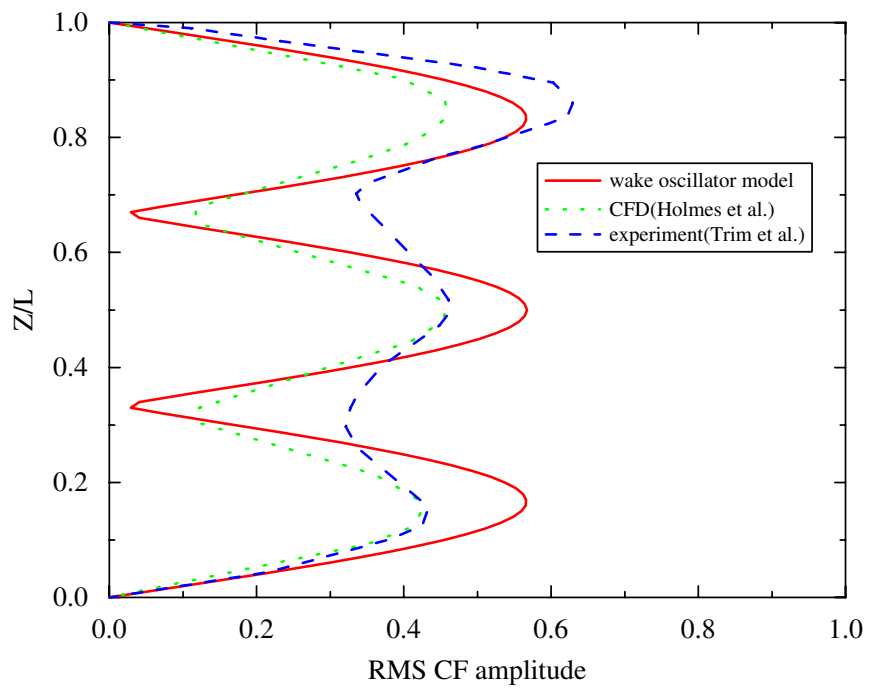

Fig. 4. Riser cross-flow (CF) VIV RMS amplitude for $U_{\text {flow }}=0.4 \mathrm{~m} / \mathrm{s}$ in uniform flow.

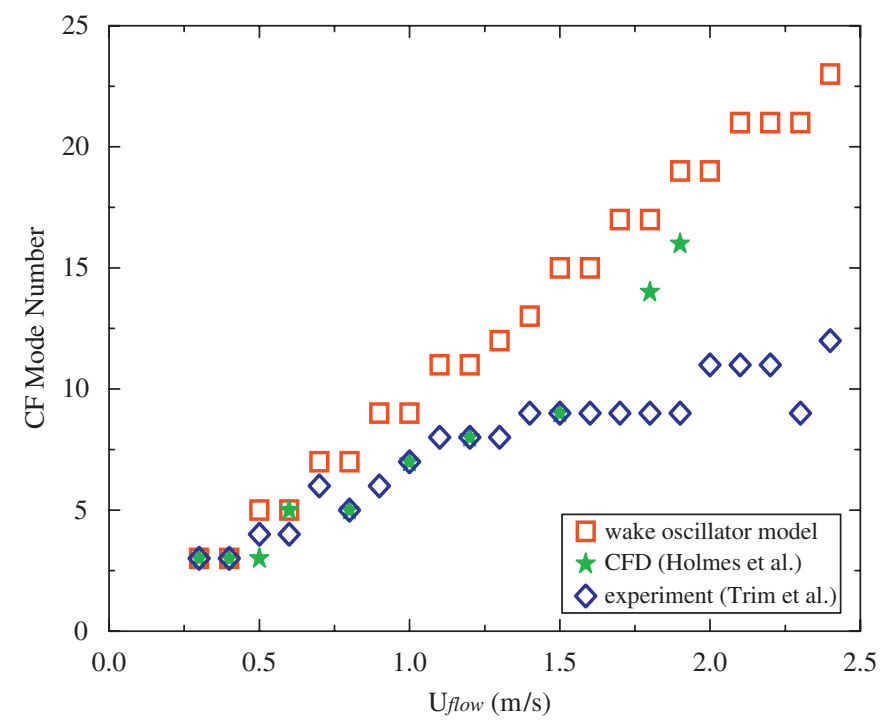

Fig. 5. Dominate cross-flow (CF) mode vs. the speed of uniform flow.

$U_{\text {flow }}=0.4 \mathrm{~m} / \mathrm{s}$. Note the asymmetry in experimental data which gives some indication of the complexity of VIV process. But the symmetry occurs in wake oscillator and CFD results, because CFD method and wake oscillator model are both simplification of actual riser VIV.

The dominating mode for different uniform flow speeds is plotted in Fig. 5. The same as CFD method, the wake oscillator model results seem to agree fairly well with the experimental data at low values of flow speed, but overestimate the mode number at high values of flow speeds. One possible reason would be the tension variation. In our calculation the tension within the riser is set to $5 \mathrm{kN}$, while in the model testing it varies in a range from 4 to $6 \mathrm{kN}$.

The mean maximum CF RMS amplitude is compared at uniform flow in Fig. 6. The CFD simulation tends to under estimate the experimental results. Since only few CFD simulations were completed at $0.4,1.5$, and $2.0 \mathrm{~m} / \mathrm{s}$ uniform flow speed, it cannot be drawn a conclusion whether the CFD method agree well with the experiment and present model or not. But present wake oscillator model shows very good agreement to the experimental data. 


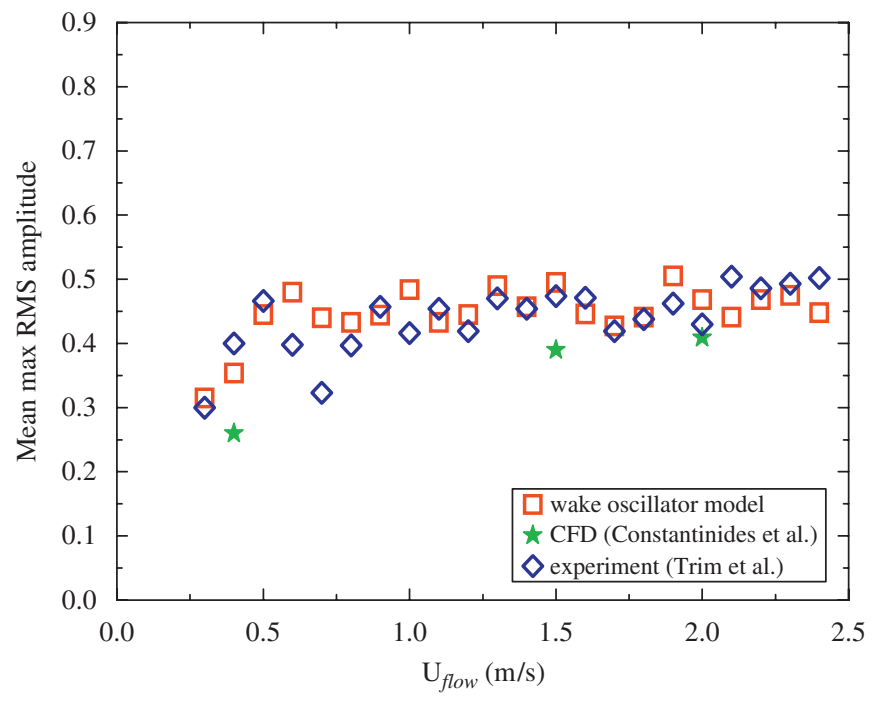

Fig. 6. Mean maximum RMS amplitude vs. the speed of uniform flow.

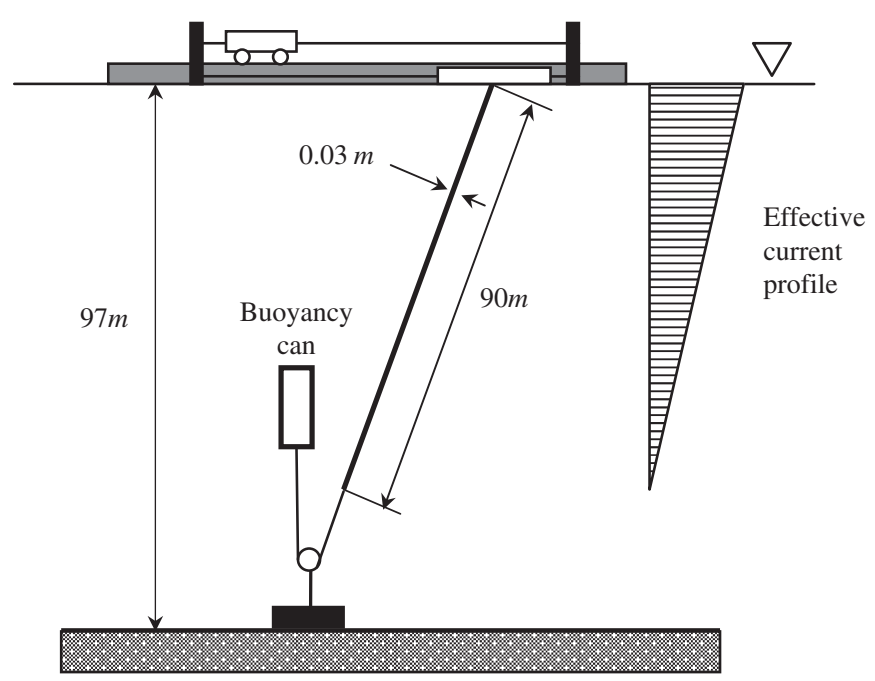

Fig. 7. Test set-up at Hanøytangen.

\subsection{Sheared flow}

Here, we only compare the predicted results with the experimental data firstly introducing the details of experiments. Tests of a tensioned large-scale riser model in well-defined sheared current were performed at Hanøytangen outside Bergen on the west coast of Norway in 1997. The test set-up is illustrated in Fig. 7. The test site is a $180 \mathrm{~m}$ long floating quay at $97 \mathrm{~m}$ depth. The length of the model was $90 \mathrm{~m}$ and the diameter was $3 \mathrm{~cm}$. The aspect ratio $L / D$ is 3000 . The riser model was attached to a floating vessel outside the quay that was pulled by a rope system and a motor vehicle on the quay. The riser model was kept at constant tension $3700 \mathrm{~N}$ by a buoyancy arrangement, as seen in the figure. More details of the riser models physical properties are shown in Table 2. By moving the vehicle at a constant speed, the riser was exposed to a triangular current profile. In this way a well-defined flow profile was obtained.

The experimental results, including the dominating mode and dimensionless mean maximum CF RMS amplitude, were found by analysis of Hanøytangen data (Lie and Kaasen, 2006). Comparing the predicted results of the riser CF motion RMS amplitude by wake oscillator model with experimental data (Lie and Kaasen, 2006) at sheared flow speed of $0.54 \mathrm{~m} / \mathrm{s}$, which is plotted in Fig. 8.
Table 2

Physical property for the riser model in sheared flow Lie and Kaasen (2006)

\begin{tabular}{ll}
\hline Total length & $90.0 \mathrm{~m}$ \\
Outer diameter & $0.03 \mathrm{~m}$ \\
Inner diameter & $0.026 \mathrm{~m}$ \\
Bending stiffness & $3.64 \times 10^{3} \mathrm{Nm}^{2}$ \\
Axial stiffness & $3.69 \times 10^{7} \mathrm{~N}$ \\
Riser density & $3211 \mathrm{~kg} / \mathrm{m}^{3}$ \\
Mass & $2.27 \mathrm{~kg} / \mathrm{m}$ \\
Mass ratio & 3.13 \\
Aspect ratio & 3000 \\
Tension at top & $3700 \mathrm{~N}$ \\
\hline
\end{tabular}

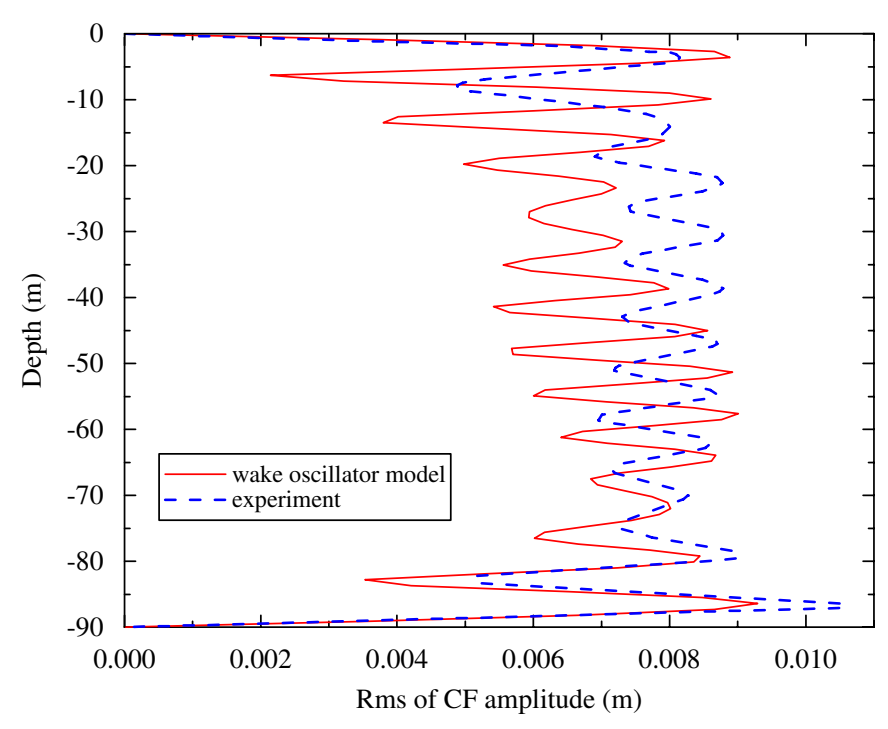

Fig. 8. Riser cross-flow (CF) VIV RMS amplitude for $U_{\text {flow }}=0.54 \mathrm{~m} / \mathrm{s}$ in sheared flow.

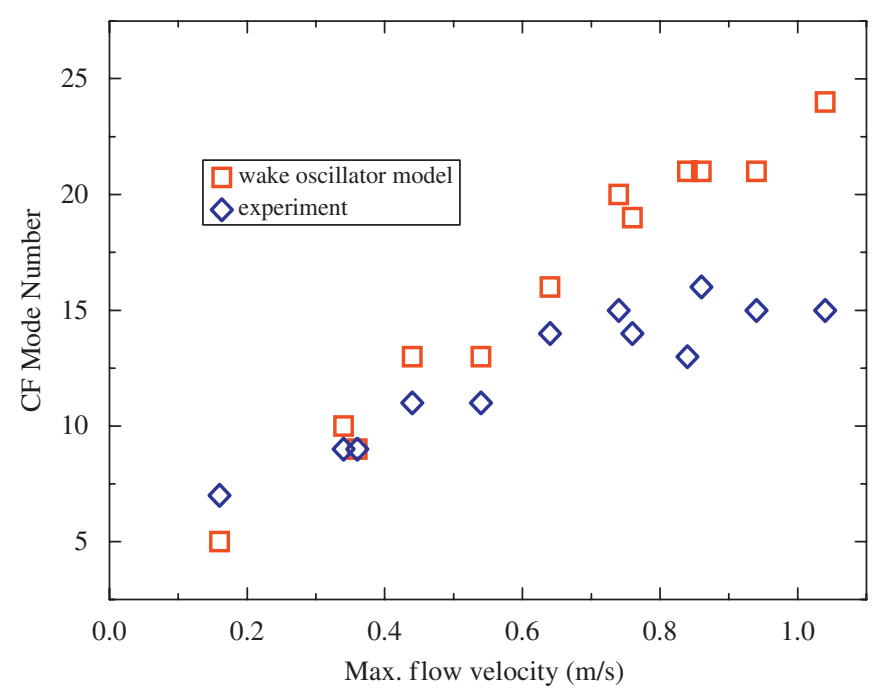

Fig. 9. Dominate cross-flow (CF) mode vs. the speed of sheared flow.

It shows that the dominant mode of wake oscillator predicted result is 13 th, while 11 th is obtained at the model testing. The maximum amplitude by experiment is a little larger than present wake oscillator predicted results.

We calculate the dominating mode for different sheared flow speeds, and compare the predicted results with the experimental data (Lie and Kaasen, 2006) in Fig. 9, the same as the case of uniform flow, the wake oscillator model results agree well with 


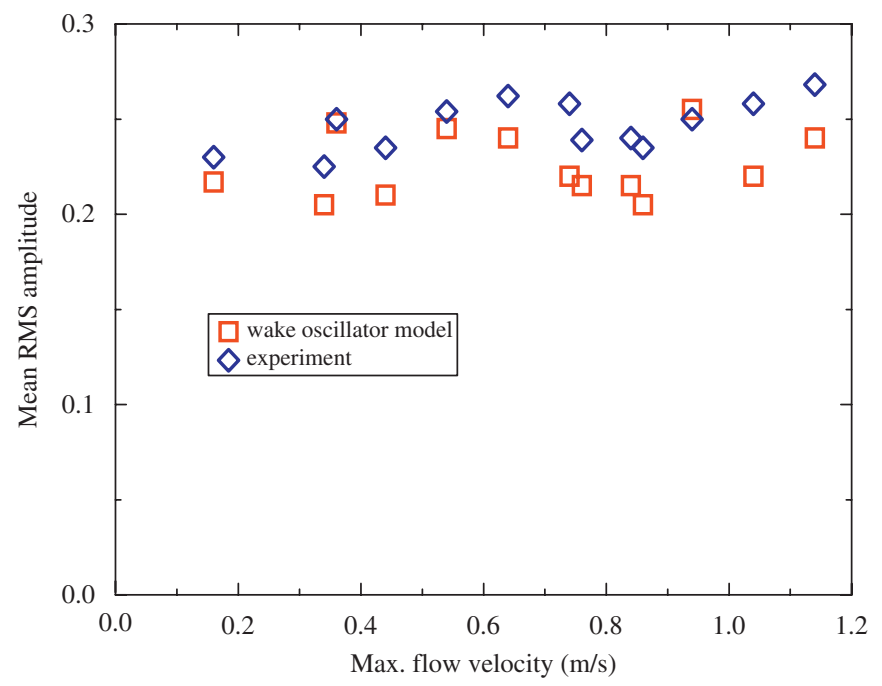

Fig. 10. Mean maximum RMS amplitude vs. the speed of sheared flow.

the experimental data at low values of flow speeds, but overestimate the mode number at high values of flow speeds. The most probable reason for this is that a fixed Strouhal number of 0.2 was used for all calculations, which might be slightly higher than the value 0.17 used by Lie and Kaasen (2006).

The mean maximum CF RMS amplitude was also calculated at sheared flow, the predicted results are compared with experimental data in Fig. 10. It can be drawn a conclusion that present predicted results show good agreement to the experimental data, and the same trend has been seen at uniform flow, see Fig. 6 .

Thus, the wake oscillator model described above gives a good picture of the successes to predicted high aspect ratio $(L / D)$ riser VIV at uniform and sheared flow.

\section{Conclusions}

The dynamics of high aspect ratio $(L / D)$ riser under VIV is very complicated. Developing a feasible approach to analyze this problem is necessary. In this paper, a simple empirical model was presented. The structural nonlinearities were considered. The van der Pol wake oscillators is used to describe the near wake. Comparisons with experimental data and CFD results have shown that the present model could predict some aspects of long flexible riser VIV. Because of the simplicity of the model, all the results presented in this paper required a small computational time, as of today, this is one of the main advantages of the wake oscillator model over the CFD method. So it is feasible to use present model undertake large parametrical studies of riser VIV which are needed for practical applications.

\section{Acknowledgments}

This research is supported by the "National High-Tech Research and Development Program (863 Program)" of China (No.
2006AA09Z350), the "National Natural Science Foundation" of China (No. 10702073) and the "Knowledge Innovation Program" of Chinese Academy of Sciences (No. KJCX2-YW-L02).

\section{References}

Baarholm, G.S., Larsen, C.M., Lie, H., 2005. Reduction of VIV using suppression devices-an empirical approach. Marine Structure 18, 489-510.

Balasubramanian, S., Skop, R.A., 1997. A new twist on an old model for vortexexcited vibrations. Journal of Fluids and Structures 11, 395-412.

Benassai, G., Campanile, A., 2002. A prediction technique for the transverse vortex-induced oscillations of tensioned risers. Ocean Engineering 29 1805-1825.

Bishop, R.E.D., Hassan, A.Y., 1964. The lift and drag forces on a circular cylinder in a flowing fluid. Proceedings of the Royal Society A 277, 32-50.

Blevins, R.D., 1990. Flow-Induced Vibrations. Van Nostrand Reinhold, New York.

Constantinides, Y., Oakley O.H., Holmes, S., 2007. CFD high L/D riser modeling study, OMAE 2007, 29151.

Facchinetti, M.L., de Langre, E., Biolley, F., 2004a. Coupling of structure and wake oscillators in vortex-induced vibrations. Journal of Fluids and Structures 19, 123-140.

Facchinetti, M.L., de Langre, E., Biolley, F., 2004b. Vortex-induced traveling waves along a cable. European Journal of Mechanics B-Fluids 23, 199-208.

Hartlen, R.T., Currie, I.G., 1970. Lift oscillation model for vortex-induced vibration. Journal of Engineering Mechanics-ASCE 96, 577-591.

Holmes, S., Oakley, O.H., Constantinides, Y., 2006. Simulation of riser VIV using fully three dimensional CFD simulations, OMAE 2006, 92124.

Huse, E., Kleiven, G., Nielsen, F.G., 1998. Large scale model testing of deep sea risers. In: Proceedings of the Offshore Technology Conference, Houston, Texas, OTC 8701.

Iwan, W.D., 1975. The vortex induced oscillation of elastic structural elements. ASCE Journal of Engineering for Industry 97, 1378-1382.

Kim, W.-J., Perkins, N.C., 2002. Two-dimensional vortex-induced vibration of cable suspensions. Journal of Fluids and Structures 16 (2), 229-245.

Koopman, G.H., 1967. The vortex wakes of vibrating cylinders at low Reynolds numbers. Journal of Fluid Mechanics 28, 501-512.

Kuchnicki, S.N., Benaroya, H., 2002. Coupled transverse and axial motion of a compliant structure in response to vortex-shedding loads. Journal of sound and vibration 257 (5), 903-929.

Lie, H., Kaasen, K.E., 2006. Modal analysis of measurements from a large-scale VIV model test of a riser in linearly sheared flow. Journal of Fluids and Structures 22, 557-575.

Lu, X.Y., Dalton, C., 1996. Calculation of the timing of vortex formation from an oscillating cylinder. Journal of Fluids and Structures 10 527-541.

Mathelin, L., de Langre, E., 2005. Vortex-induced vibrations and waves under shear flow with a wake oscillator model. European Journal of Mechanics B-Fluids $24,478-490$.

Nayfeh, A.H., 1993. Introduction to Perturbation Techniques. Wiley, New York.

Shulz, K.W., Meling, T.S., 2004. Multi-strip numerical analysis for flexible riser response, OMAE 2004, 51186

Shulz, K.W., Meling, T.S., 2005. VIV analysis of a riser subjected to step and multidirectional currents, OMAE 2005, 67144.

Skop, R.A., Griffin, O.M., 1973. A model for the vortex-excited resonant response of bluff cylinders. Journal of Sound and Vibration 27 (2), 225-233.

Skop, R.A., Luo, G., 2001. An inverse-direct method for predicting the vortexinduced vibrations of cylinders in uniform and nonuniform flows. Journal of Fluids and Structures 15, 867-884.

Stansby, P.K., 1976. The locking-on of vortex shedding due to the cross-stream vibration of circular cylinders in uniform and shear flows. Journal of Fluid Mechanics 74, 641-665.

Trim, A.D., Braaten, H., Lie, H., Tognarelli, M.A., 2005. Experimental investigation of vortex-induced vibration of long marine risers. Journal of Fluids and Structures 21, 335-361.

Violette, R., de Langre, E., Szydlowski, J., 2007. Computation of vortex-induced vibrations of long structures using a wake oscillator model: comparison with DNS and experiments. Computers \& Structures 85, 1134-1141.

Willden, R.H.J., Graham, J.M.R., 2005. CFD simulations of the vortex-induced vibrations of model riser pipes. OMAE 2005, 67197.

Yamamoto, C.T., Meneghini, J.R., Saltara, F., Fregonesi, R.A., Ferrari Jr., J.A., 2004. Numerical simulations of vortex-induced vibration on flexible cylinders. Journal of Fluids and Structures 19, 467-489. 